\title{
HYPERSPECTRAL TRANSFORMATION FROM EO-1 ALI IMAGERY USING PSEUDO-HYPERSPECTRAL IMAGE SYNTHESIS ALGORITHM
}

\author{
Nguyen Tien Hoang ${ }^{\mathrm{a}, \mathrm{b}}$, Katsuaki Koike $\mathrm{a}^{\mathrm{a}, *}$ \\ ${ }^{\text {a }}$ Graduate School of Engineering, Kyoto University, Katsura C1-2-215, Kyoto 615-8540, Japan - koike.katsuaki.5x@ kyoto-u.ac.jp \\ ${ }^{\mathrm{b}}$ Department of Environmental Science, College of Sciences, Hue University, 77 Nguyen Hue, Hue, Vietnam - \\ nguyenhoanggis@gmail.com
}

Commission VII, WG VII/6

KEY WORDS: Hyperspectral data, multiple analysis, spectral reconstruction, Hyperion, ALI

\begin{abstract}
:
Hyperspectral remote sensing is more effective than multispectral remote sensing in many application fields because of having hundreds of observation bands with high spectral resolution. However, hyperspectral remote sensing resources are limited both in temporal and spatial coverage. Therefore, simulation of hyperspectral imagery from multispectral imagery with a small number of bands must be one of innovative topics. Based on this background, we have recently developed a method, Pseudo-Hyperspectral Image Synthesis Algorithm (PHISA), to transform Landsat imagery into hyperspectral imagery using the correlation of reflectance at the corresponding bands between Landsat and EO-1 Hyperion data. This study extends PHISA to simulate pseudo-hyperspectral imagery from EO-1 ALI imagery. The pseudo-hyperspectral imagery has the same number of bands as that of high-quality Hyperion bands and the same swath width as ALI scene. The hyperspectral reflectance data simulated from the ALI data show stronger correlation with the original Hyperion data than the one simulated from Landsat data. This high correlation originates from the concurrent observation by the ALI and Hyperion sensors that are on-board the same satellite. The accuracy of simulation results are verified by a statistical analysis and a surface mineral mapping. With a combination of the advantages of both ALI and Hyperion image types, the pseudo-hyperspectral imagery is proved to be useful for detailed identification of minerals for the areas outside the Hyperion coverage.
\end{abstract}

\section{INTRODUCTION}

Recent advances in hyperspectral remote sensing (also known as imaging spectroscopy) have demonstrated that hyperspectral imagery is more effective than multispectral imagery in many application fields because of having hundreds of observation bands with high spectral resolution. However, hyperspectral remote sensing resources are limited both in temporal and spatial coverage. Therefore, simulation of hyperspectral imagery from multispectral imagery with a small number of bands must be one of innovative topics.

The National Aeronautics and Space Administration EO-1 satellite was successfully launched on November 21, 2000. EO-1 brings three sensors including the multispectral Advanced Land Imager (ALI), the hyperspectral Hyperion sensor, and the Linear Etalon Imaging Spectrometer Array (LEISA) Atmospheric Corrector (LAC). EO-1 Hyperion, a representative space-based imaging spectroscopy, enables a wide range of applications, including mining, geology, forestry, agriculture and environmental management. Hyperion covers the $0.4-2.5 \mu \mathrm{m}$ wavelength range with 242 bands at approximately $10 \mathrm{~nm}$ spectral resolution and 30 $\mathrm{m}$ spatial resolution. Despite this spectral superiority, its image scene is narrower than ALI image scene (Table 1). In addition, ALI data are much less noise than Hyperion data. If ALI imagery can be successfully transformed into Hyperion data, this pseudohyperspectral imagery must be more helpful because of a combination of the advantages of both image types. Since 2008 some researchers have addressed the simulation of Hyperion data from ALI images. Chen et al. (2008) used a model of spectrum mixing based on spectral library and Liu et al. (2009) used the universal pattern decomposition method (UPDM) to acquire simulated hyperspectral images. Both their studies have only focused on ac-

\footnotetext{
${ }^{*}$ Corresponding author
}

quiring pseudo-hyperspectral images inside the area covered by original hyperspectral image scenes.

We have recently developed a new method, Pseudo-Hyperspectral Image Synthesis Algorithm (PHISA), to transform Landsat ETM+ imagery into hyperspectral imagery using the correlation of reflectance at the corresponding bands between ETM+ and Hyperion data (Hoang and Koike, 2015). This study extends PHISA to simulate the pseudo-hyperspectral imagery from EO-1 ALI imagery. The pseudo-hyperspectral imagery can have the number of bands with the same number as high-quality Hyperion bands and with the same swath width as ALI scene.

\section{DATA AND STUDY AREA}

We used three cloud-free images acquired on 23 July 2001, EO-1 ALI, EO-1 Hyperion and Landsat 7 ETM+ images, which were obtained from USGS Earth Explorer. Figure 1 illustrates the overlap in surface area coverage of the ALI and Hyperion scenes, compared to the Landsat 7 ETM+ ground track. EO-1 flies approximately one minutes behind Landsat 7 with the same sunsynchronous orbit at an altitude of $705 \mathrm{~km}$. Because PHISA was originally developed for Landsat data, the ETM+ imagery was

\begin{tabular}{|l|l|l|}
\hline Parameters & ALI & EO1-Hyperion \\
\hline Spectral range & $0.4-2.4 \mu \mathrm{m}$ & $0.4-2.5 \mu \mathrm{m}$ \\
Spectral resolution & Variable & $\approx 10 \mathrm{~nm}$ \\
Spectral coverage & Discrete & Continuous \\
Number of bands & 10 & 220 \\
Swath width & $37 \mathrm{~km}$ & $7.5 \mathrm{~km}$ \\
Spatial resolution & $30 \mathrm{~m}$, PAN: $10 \mathrm{~m}$ & $30 \mathrm{~m}$ \\
Temporal resolution & Variable & Variable \\
\hline
\end{tabular}

Table 1: ALI and Hyperion technical specifications 


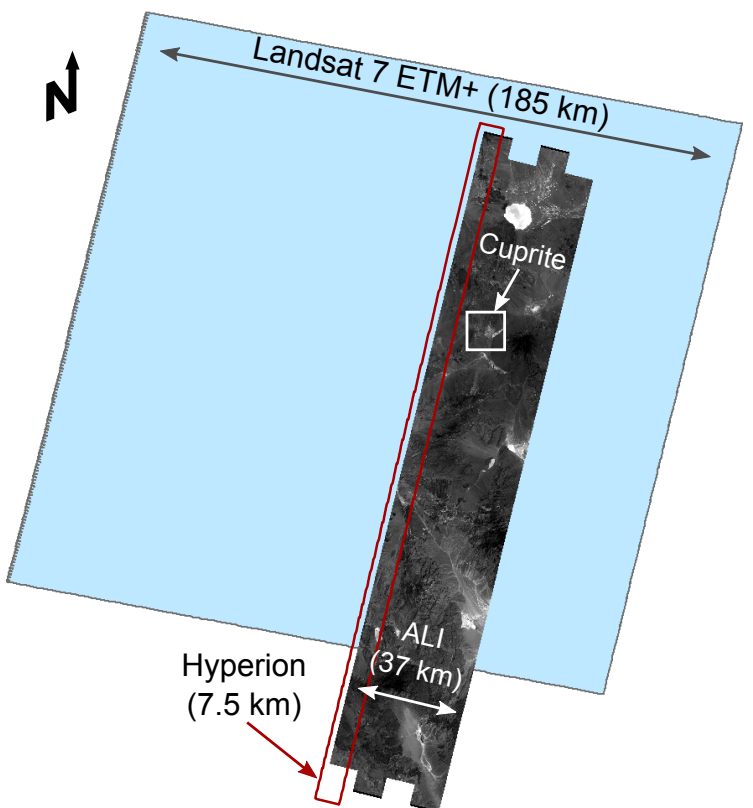

Figure 1: Locations of EO1-Hyperion, ALI and Landsat 7 ETM+ scenes used in this study

\begin{tabular}{|c|c|c|}
\hline Band & Wavelength $(\mu \mathrm{m})$ & Description \\
\hline PAN & $0.48-0.69$ & Panchromatic \\
MS-1' & $0.433-0.453$ & VNIR (blue) \\
MS-1 & $0.45-0.515$ & VNIR (blue) \\
MS-2 & $0.525-0.605$ & VNIR (green) \\
MS-3 & $0.63-0.69$ & VNIR (red) \\
MS-4 & $0.775-0.805$ & VNIR \\
MS-4' & $0.845-0.890$ & VNIR \\
MS-5' & $1.2-1.3$ & SWIR \\
MS-5 & $1.55-1.75$ & SWIR \\
MS-7 & $2.08-2.35$ & SWIR \\
\hline
\end{tabular}

Table 2: Spectral characteristics for the ALI bands

used to simulate the pseudo-hyperspectral data for comparison with the one simulated from ALI. The spectral characteristics of ALI bands are summarized in Table 2. In the VNIR and SWIR spectral ranges, ALI image consists 9 multispectral bands versus 6 bands of ETM+ image (except for the panchromatic band). All images are cloud-free and located in an area lying on the border of California and Nevada in US (path 41 and row 34).

The ALI scene covers the Cuprite alteration zones while the Hyperion scene does not cover this area. Therefore, Cuprite was chosen for validation of the pseudo-hyperspectral data outside the Hyperion scene. With an extremely arid climate condition, the Cuprite site is barren and sparsely vegetated land, which is suitable for remote sensing-based mineral mapping. Cuprite served as the test site of many remote sensing instruments including airborne and orbital visible, near-infrared, thermal-infrared, and hyperspectral sensors (Swayze et al., 2014). To further evaluate PHISA, we selected the AVIRIS image as a ground truth data collected on July 12, 2002 for mineral mapping. The AVIRIS data having a spatial resolution of 15.7 meters was provided in an orthocorrected radiance data format by Jet Propulsion Laboratory. The mineral map for the Cuprite site built from the pseudohyperspectral data was validated by a map classified from the AVIRIS data.



Figure 2: Flow chart of the PHISA method

\section{METHODS}

\subsection{PHISA}

Every reflectance spectra of surface materials usually follow certain rules or "behaviours" in which a reflectance value at a channel can be predicted from reflectance values of "key" channels. Based on this idea, PHISA has been developed by assuming that multiple linear regression models can be hold between each of Hyperion bands and Landsat ETM+ bands, in which each Hyperion band is a response variable and Landsat bands are predictor variables (Hoang and Koike, 2015). In this study, we used ALI bands as substitutes of Landsat bands, and then the general form of the multiple linear regression is defined as

$$
\begin{aligned}
H_{i j}= & \beta_{0 i}+\beta_{1 i} \cdot A_{1 j}+\beta_{2 i} \cdot A_{2 j}+\beta_{3 i} . A_{3 j}+\beta_{4 i} \cdot A_{4 j}+\beta_{5 i} . \\
& A_{5 j}+\beta_{6 i} . A_{6 j}+\beta_{7 i} \cdot A_{7 j}+\beta_{8 i} . A_{8 j}+\beta_{9 i} \cdot A_{9 j}+\varepsilon_{i j},
\end{aligned}
$$

where $H_{i j}$ represents pixel value of Hyperion image at band $i$ and location $j ; \beta_{0 i}$ is intercept at Hyperion band $i ; \beta_{1 i}, \beta_{2 i}, \beta_{3 i}, \beta_{4 i}$, $\beta_{5 i}, \beta_{6 i}, \beta_{7 i}, \beta_{8 i}$, and $\beta_{9 i}$ are unknown regression coefficients between ALI bands and Hyperion band $i ; A_{1 j}, A_{2 j}, A_{3 j}, A_{4 j}$, $A_{5 j}, A_{6 j}, A_{7 j}, A_{8 j}$, and $A_{9 j}$ represent pixel values at location $j$ 

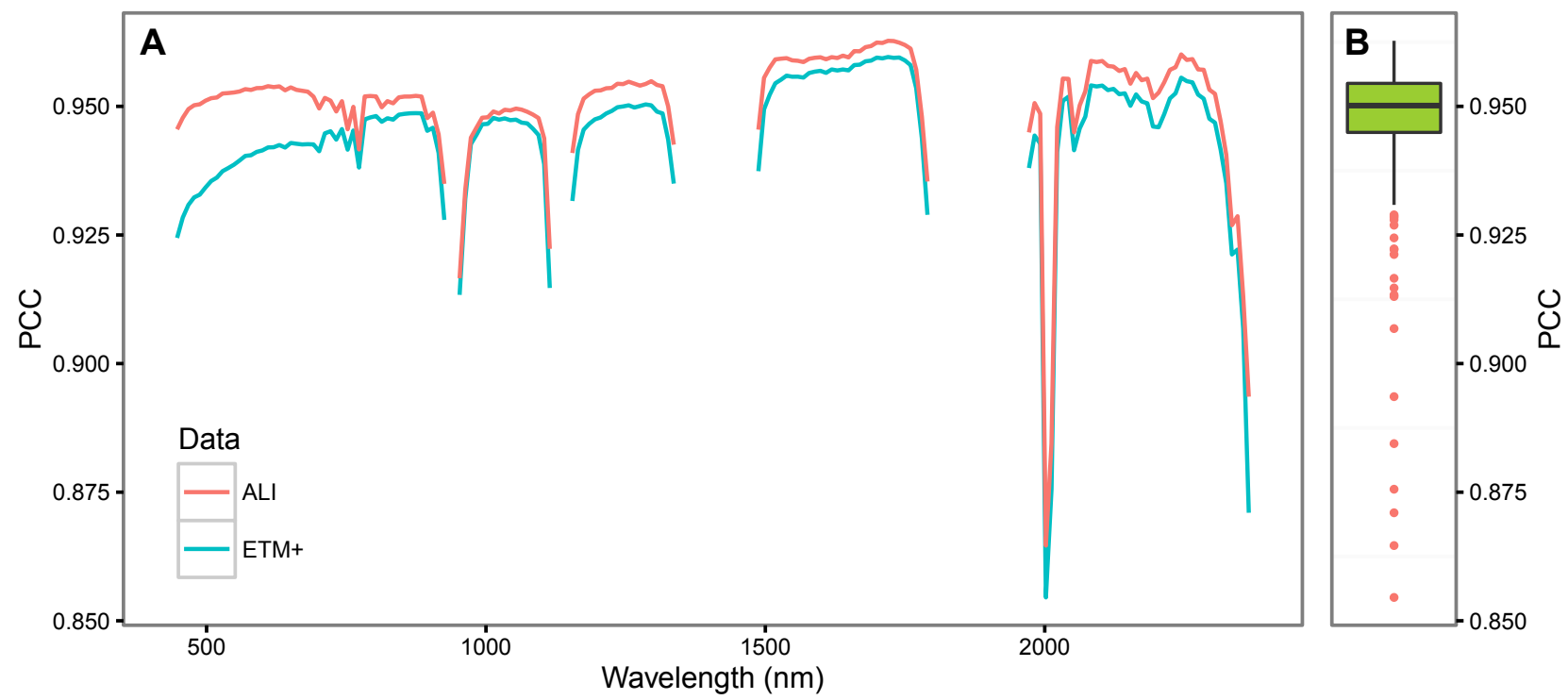

Figure 3: (A) Comparison between two cases of the pseudo-hyperspectral data simulated from ALI and Landsat ETM+ images for PCCs of the original Hyperion data and the pseudo-hyperspectral data; (B) Boxplot of PCCs between the original Hyperion data and the ALI based pseudo-hyperspectral data for 155 simulated bands

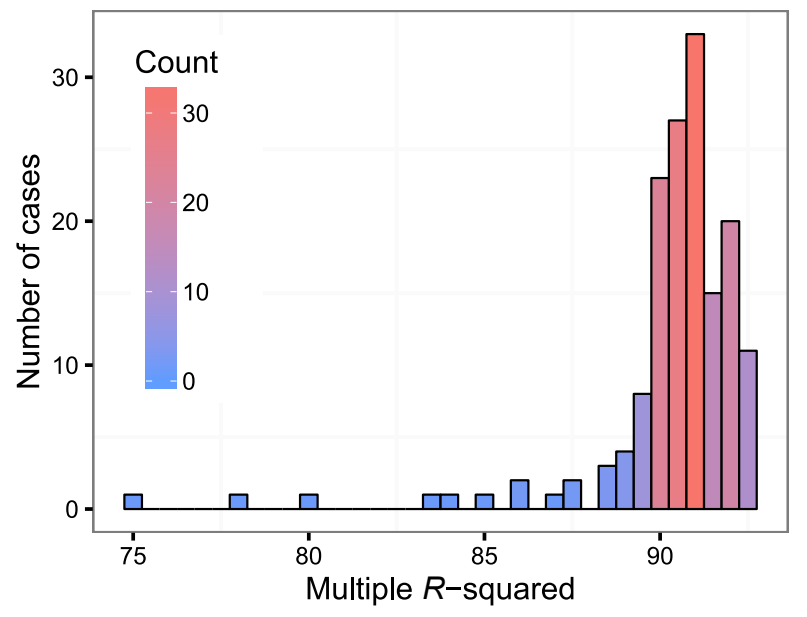

Figure 4: Histogram of multiple $R$-squared

of ALI Band 1', 1, 2, 3, 4, 4', 5', 5, and 7, respectively; and $\varepsilon_{i j}$ is random error (residual) at band $i$ and location $j$.

Bayesian model averaging (BMA) method is applied to correctly determine the relevant ALI bands in every Hyperion band regenerating models. The variable selection was done using BMA package in $\mathrm{R}$ programming language. The best model, which was selected from a set of possible models, has the lowest Bayesian Information Criterion (BIC) and the highest posterior probability (Raftery et al., 2005). This model is used to build the corresponding pseudo-hyperspectral band which has the same swath width as the ALI scene. Flow chart of the PHISA method is shown in Figure 2.

Hyperion data suffer from systematic and random noise which was reduced prior to further processing. Accordingly, the Hyperion image was corrected at first for smile effects by the moving linear fitting and interpolation method (Goodenough et al., 2003), and then, for vertical strips at outlier pixels by the local de-striping method (Datt et al., 2003). The ENVI MODTRAN- based Fast Line-of-sight Atmospheric Analysis of Spectral Hypercubes (FLAASH) module was used for atmospheric correction and producing surface reflectance images from both ALI and Hyperion data. The C-correction method, a non-Lambertian technique, was chosen for topographic correction. Hyperion scene was co-registered to ALI scene. Only the overlapped area of ALI and Hyperion data were used to build the model dictionary.

\subsection{Validation}

The quality of the pseudo-hyperspectral data are verified by statistical analysis and an application of mineral mapping. Two statistical metrics used in the validation are Pearson's correlation coefficient (PCC) and the root mean square error (RMSE) between the original Hyperion data and the pseudo-hyperspectral data. For mineral mapping, a hyperspectral data analysis approach implemented in the ENVI software, Spectral Hourglass Wizard, was used for both pseudo-hyperspectral and AVIRIS data. The details of this technique are described in Kruse et al. (2003).

\section{RESULTS AND DISCUSSION}

\subsection{Statistical analysis}

The accuracy of each multiple linear regression model between Hyperion and ALI bands was confirmed by a high coefficient of determination (multiple $R$-squared). Most models had the multiple $R$-squared higher than $90 \%$ (Figure 4 ). The highest, mean and lowest values are $92.7 \%, 90.4 \%$ and $74.8 \%$, respectively. Some models include all multispectral bands of ALI imagery but some have only 6 bands as predictor variables. Band 2 of ALI imagery appears the most frequently (155 times), while Band 4' is less frequently observed (128 times) in the models. However, we are of the opinion that these frequencies may be changed according to study area because types and area ratios of surface materials are different.

Since all multiple linear regression models achieved high accuracies, ALI imagery was transformed into 155 bands of the pseudohyperspectral imagery. The most remarkable result to emerge 

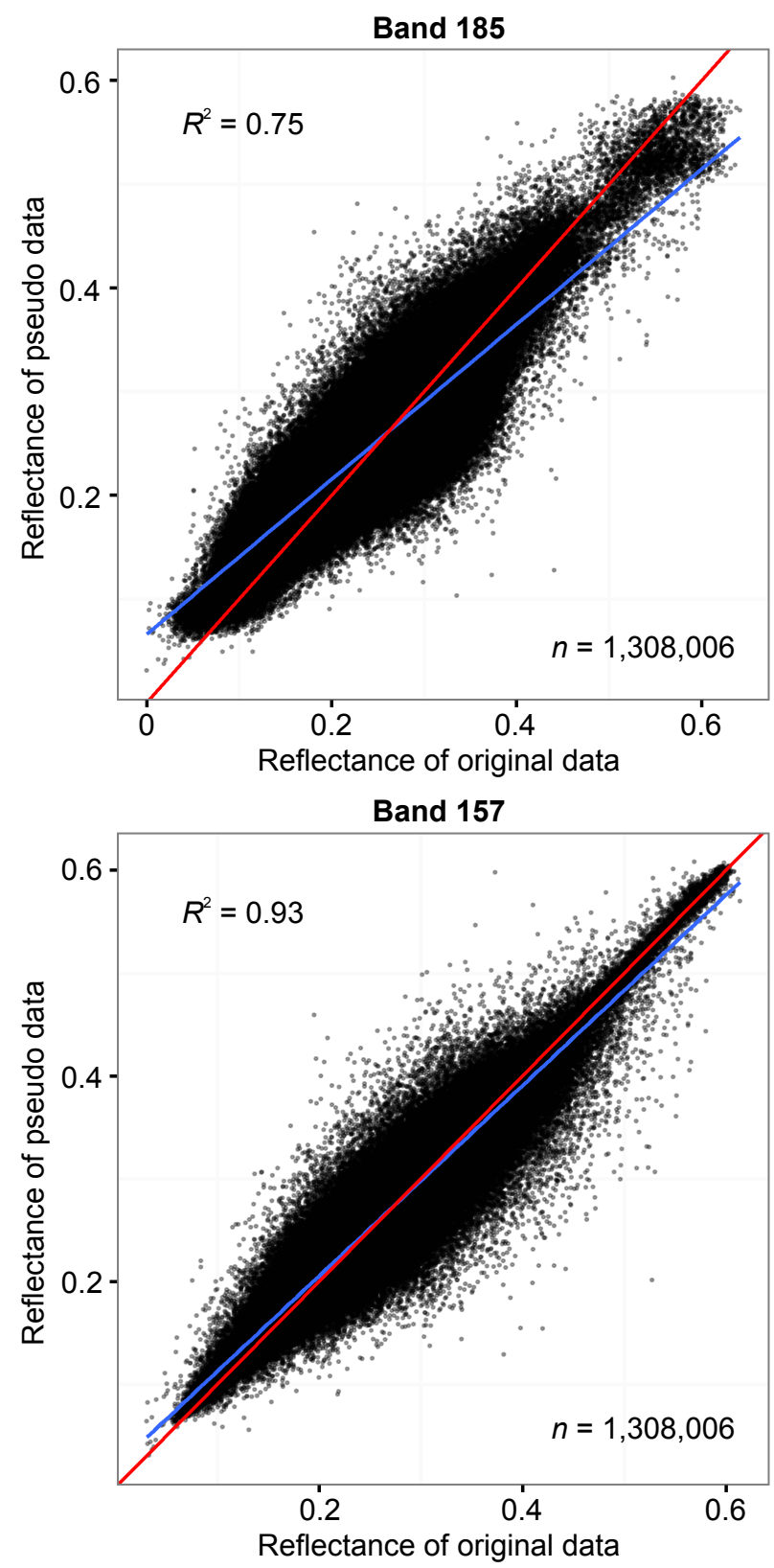

Figure 5: Examples of linear regression between the original Hyperion data and the pseudo-hyperspectral data at Bands 157 and 185

from the statistical analysis is that most bands have PCCs > 0.95 . Only a small fraction of pseudo-bands has the coefficients $<0.93$ like outliers in the dataset (Figure 3B). Even the lowest correlation coefficient was still high at 0.86 . In addition, the RMSE values, which are consistent with PCCs, are mainly low, smaller than 0.016. Figure 3A shows plots of PCCs between the original Hyperion data and the pseudo-hyperspectral data for two cases of the pseudo-hyperspectral data simulated from ALI and Landsat ETM+ images. It is noteworthy that the curve of ALI based pseudo-data is above that of the Landsat based pseudodata, which means the pseudo-hyperspectral data simulated from the ALI data is more strongly correlated with the original Hyperion data than the one simulated from Landsat data. This high correlation originates from the concurrent observation by the ALI and Hyperion sensors that are on-board the same satellite.

The results revealed consistent agreements between the original
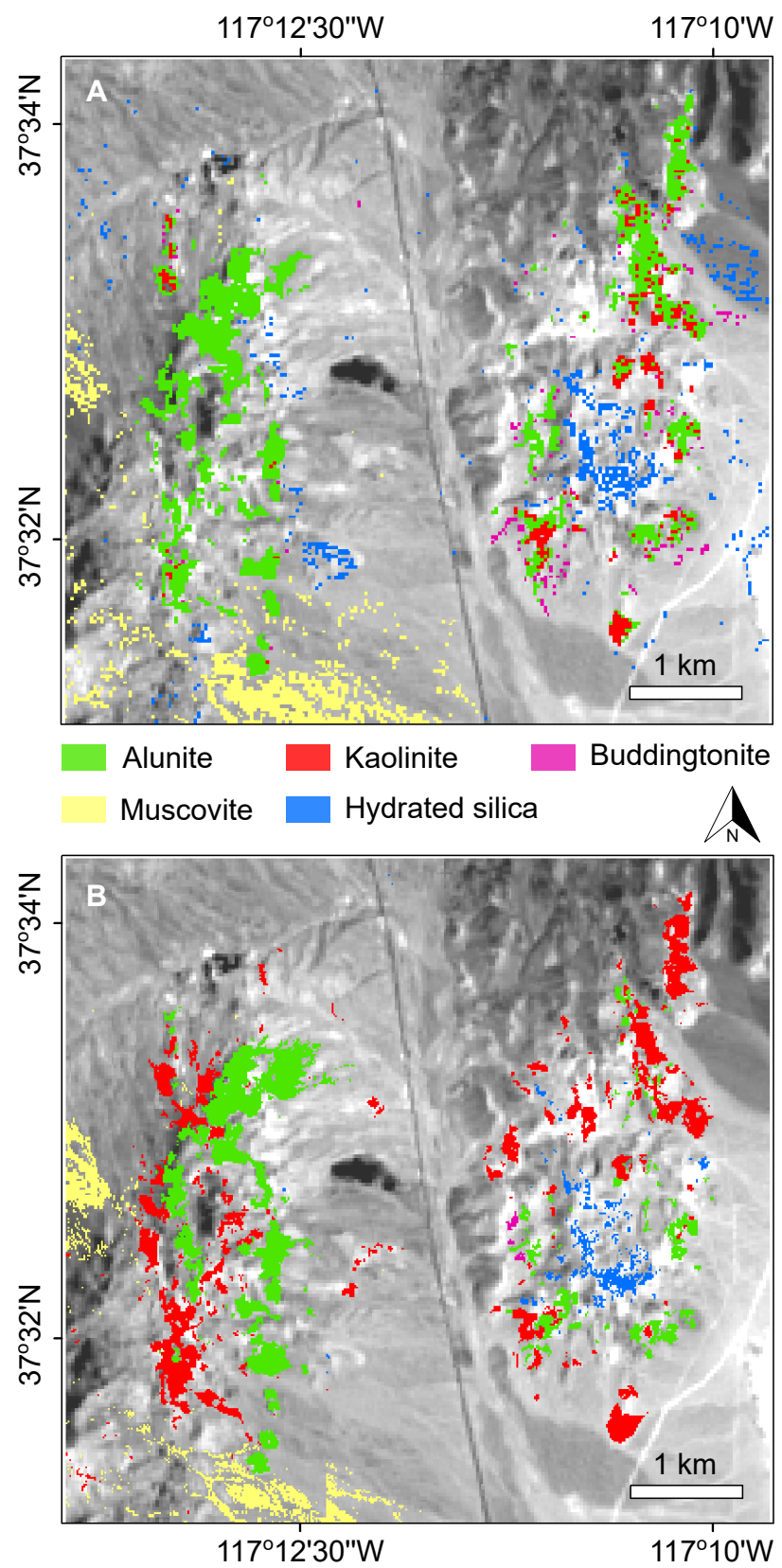

Figure 6: Mineral maps of spectrally dominant selected endmembers for the pseudo-hyperspectral data (A) and AVIRIS (B). Background is grayscale of the pseudo-hyperspectral band 190

Hyperion data and the pseudo-hyperspectral data, with correlation slopes close to one (Figure 5). Each coefficient of determination was calculated by using all pixel values of the corresponding band. The highest linearity $\left(R^{2}=0.93\right)$ that was identified in the case of Band 157 confirmed the strong similarity of the pseudo-data to the original data for this band. Even the lowest coefficient of determination was still high in the case of Band $185\left(R^{2}=0.75\right)$. Those observations suggest the statistical suitability of PHISA for transforming ALI imagery into the pseudohyperspectral imagery.

\subsection{Mineral mapping}

Spectral bands covering the SWIR ranges $(2.0-2.4 \mu \mathrm{m})$ of the pseudo-hyperspectral and AVIRIS data were selected for mineral mapping. The pseudo-data was co-registered and resampled to AVIRIS data. Five basic mineral endmembers, which consists of 


\begin{tabular}{|l|c|c|c|c|c|}
\cline { 2 - 6 } \multicolumn{1}{c|}{} & \multicolumn{5}{c|}{ AVIRIS Ground Truth (\%) } \\
\hline Pseudo-data endmembers & Alunite & Kaolinite & Muscovite & Buddingtonite & Hydrated silica \\
\hline Alunite & $\mathbf{8 9 . 4 0}$ & 81.35 & 0.08 & 0.00 & 0.00 \\
\hline Kaolinite & 9.54 & $\mathbf{1 6 . 8 4}$ & 0.00 & 0.00 & 0.00 \\
\hline Muscovite & 0.00 & 0.06 & $\mathbf{9 9 . 9 2}$ & 0.00 & 0.00 \\
\hline Buddingtonite & 1.03 & 1.75 & 0.00 & $\mathbf{1 0 0 . 0 0}$ & 0.00 \\
\hline Hydrated silica & 0.03 & 0.00 & 0.00 & 0.00 & $\mathbf{1 0 0 . 0 0}$ \\
\hline
\end{tabular}

Table 3: Confusion matrix comparing the mineral mapping results between pseudo-hyperspectral and AVIRIS images

kaolinite, alunite, muscovite, hydrated silica (chalcedony and/or opal), and buddingtonite, were determined based on the result of purest pixels in n-dimensional space (n-D Visualizer). Spectral Angle Mapper (SAM) method was used to produce distribution and abundance maps of selected minerals.

One ALI band in the $2.2 \mu \mathrm{m}$ region was transformed into 37 bands of the pseudo-hyperspectral data. This result is very important to identify mineralogical composition by exploiting absorptions found in the SWIR region. The mineral maps show both satisfactory and unexpected results (Figure 6). Muscovite and hydrated silica were well identified from the pseudo-data while most pixels classified by AVIRIS as kaolinite were misclassified as alunite on the pseudo-data. It is interesting to note that buddingtonite that isolated into small areas can be extracted from the pseudo-data. The confusion matrix revealed that there were two high classification errors in alunite mapped by the pseudo-data as kaolinite $(9.54 \%)$ and kaolinite mapped by the pseudo-data as alunite $(81.35 \%)$ (Table 3$)$. Despite the fact that the classification accuracy of muscovite, hydrated silica, and buddingtonite were $99.9 \%, 100 \%$, and $100 \%$, respectively, the kaolinite classification error declined the overall agreement of the pseudo-data with AVIRIS fall to $63 \%$. This requires further improvements of PHISA to separate endmembers having similar reflectance spectra.

\section{CONCLUSIONS}

We applied PHISA to transform EO-1 ALI imagery into 155 bands of the pseudo-hyperspectral imagery and produced the pseudodata with the same swath width as ALI scene. Most pseudo-bands have PCCs bigger than 0.95 and RMSE values smaller than 0.016 . The strong similarities between each band data of Hyperion and the pseudo-hyperspectral reflectances have further strengthened our confidence in extending applications of PHISA. Despite the statistical suitability and very high classification accuracy of muscovite, hydrated silica, and buddingtonite, the mineral mapping result showed that kaolinite were mostly misclassified as alunite. Future work should concentrate on improving PHISA by assigning the best model to each surface pattern over all bands. We believe that the improvement can reduce the unexpected performance by separating endmembers having similar reflectance spectra.

\section{ACKNOWLEDGEMENTS}

We are grateful to USGS and NASA for providing us with raw EO-1 Hyperion, ALI, Landsat 7 ETM+ and AVIRIS images.

\section{REFERENCES}

Chen, F., Niu, Z., Sun, G., Wang, C. and Teng, J., 2008. Using low-spectral-resolution images to acquire simulated hyperspectral images. International Journal of Remote Sensing, 29(10), pp. 2963-2980.
Datt, B., McVicar, T., Van Niel, T., Jupp, D. and Pearlman, J., 2003. Preprocessing EO-1 Hyperion hyperspectral data to support the application of agricultural indexes. IEEE Transactions on Geoscience and Remote Sensing, 41(6), pp. 1246-1259.

Goodenough, D. G., Dyk, A., Niemann, K. O., Pearlman, J. S., Chen, H., Han, T., Murdoch, M. and West, C., 2003. Processing Hyperion and ALI for forest classification. IEEE Transactions on Geoscience and Remote Sensing, 41(6), pp. 1321-1331.

Hoang, N. T. and Koike, K., 2015. Development of Bayesianbased transformation method of Landsat imagery into pseudohyperspectral imagery. In: Proc. SPIE 9643, Image and Signal Processing for Remote Sensing XXI, 96430J, pp. 96430J-196430J-6.

Kruse, F. A., Boardman, J. W. and Huntington, J. F., 2003. Comparison of Airborne Hyperspectral Data and EO-1 Hyperion for Mineral Mapping. IEEE Transactions on Geoscience and Remote Sensing, 41(6), pp. 1388-1400.

Liu, B., Zhang, L., Zhang, X., Zhang, B. and Tong, Q., 2009. Simulation of EO-1 Hyperion Data from ALI Multispectral Data Based on the Spectral Reconstruction Approach. Sensors, 9(4), pp. 3090-3108.

Raftery, A. E., Painter, I. S. and Volinsky, C. T., 2005. BMA: An R package for Bayesian Model Averaging. R News, 5(2), pp. 2-8.

Swayze, G. A., Clark, R. N., Goetz, A. F. H., Livo, K. E., Breit, G. N., Kruse, F. A., Sutley, S. J., Snee, L. W., Lowers, H. A., Post, J. L., Stoffregen, R. E. and Ashley, R. P., 2014. Mapping Advanced Argillic Alteration at Cuprite, Nevada, Using Imaging Spectroscopy. Economic Geology, 109(5), pp. 1179-1221. 